\title{
Les Archives Photographiques Notman du Musée McCord
}

\author{
by Barbara M. Syrek
}

Parmi les collections du Musée McCord d'histoire canadienne, il y en a une particulièrement bien connue, "Les Archives photographiques Notman". Il faut dire que la ville de Montréal a été privilégiée de se voir choisie par celui qui a créé un des plus importants studios photographiques au Canada, 17 ans après la découverte de la photographie. C'est à la fin de 1856 que William Notman, un écossais, ouvre un studio temporaire quelques mois après son arrivée à Montréal. Au fil des années, son premier studio se transforme en une grande entreprise photographique familiale.

L'importance du travail de William Notman, de ses fils et de leurs employés est basée sur le caractère de la photographie qui nous donne des images fidèles. Celle-ci est une source indubitable d'information sur le temps passé par rapport aux changements de la vie d'aujourd'hui et celle de l'avenir. L'architecture, les costumes, les voitures et même notre physionomie ne seront plus les mêmes avec le temps. Prenons en exemple les nombreux portraits de Montréal qui change continuellement pour le mieux ou le pire. On bâtit, on détruit... . Les photographies seront toujours les témoins de notre histoire.

L'année 1956 souligne le centième anniversaire de l'établissement à Montréal de William Notman, "photographer to the Queen" et surtout photographe de la vie quotidienne de la ville pendant la deuxième moitié du XIXe siècle. Cette même année marque le début de la vie publique de la "Collection Notman" qui trouve sa place aux McGill University Museums. C'est grâce à Alice J. Tumham, directrice des McGill University Museums à l'époque, et son action remarquablement persévérante auprès des autorités de l'Université, qu'on peut encore aujourd'hui admirer la richesse du passé véhiculée par la "Collection Notman" et, en plus, se servir de cette source historiquement importante.

Of all the collections in the McCord Museum of Canadian History, the Notman Photographic Archives are perhaps the best known. Montreal had the great good fortune of being the chosen home of the man who set up one of Canada's largest photography studios just 17 years after the discovery of the photographic process. William Notman arrived in Montreal from Scotland in 1856 and opened a makeshift studio a few months later. Over the years, that studio would grow to become a large family-run photography firm.

What makes the work of William Notman, his sons and their employees so important is the nature of the photography that has passed such faithful images down to us, providing a reliable source of information on times gone by to contrast with the changes that we see today and those we anticipate for tomorrow. Our architecture, costumes, automobiles, our very physiognomy - all change over time, as witness the many portraits of Montreal, a city that is continually changing, for better or for worse. Buildings go up, buildings come down... and photography continues to bear witness to our history.

The year 1956 marked the centenary of the arrival in Montreal of William Notman, "photographer to the Queen," but even more important, the photographer of daily life in this city in the second half of the nineteenth century. That milestone year saw the public debut of the Notman Collection, which found its rightful place in the McGill University Museums. It is thanks to the remarkable persistence of Alice J. Turnham, then the director of the McGill University Museums, who persuaded the university authorities that Notman's work must be preserved, that today we can admire the rich heritage passed down to us in the Notman Collection, and benefit from this historically important source. 


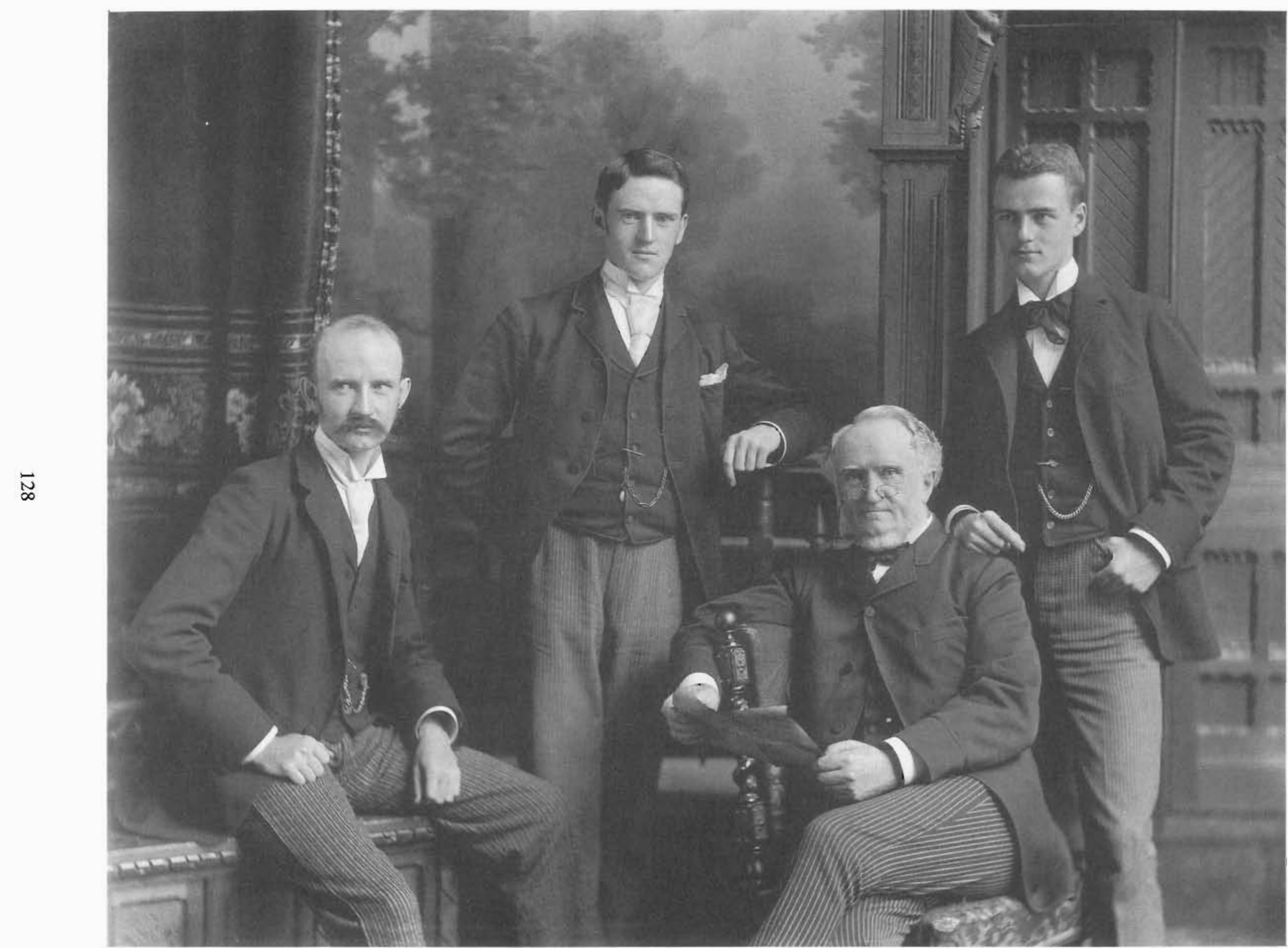

के

William Notman et ses fils William McF., George et Charles, 1890.

(Musée McCord d'histoire Canadienne, Archives photographiques Notraan' 


\section{Les Archives photographiques Notman du Musée McCord}

$\mathrm{L}$

'importance de William Notman pour le développement de la photographie au Canada est reconnue. Son nom se retrouve dans plusieurs publications professionnelles parues à travers le monde. On le connaît comme un grand portraitiste et un innovateur qui a su allier dans son travail, à la fois, une approche en tant qu'artiste et une autre comme hormme d'affaires.' Grâce à sa bonne habitude de tenir les régistres de son travail et aussi grâce aux circonstances, une grande partie de son oeuvre peut être appréciée cent ans après sa mort.

\section{LA "COLLECTION NOTMAN"; SON CHEMINEMENT VERS LE MUSÉE MCCORD.}

La succession de William Notman se retrouve aujourd'hui aux Archives photographiques Notman du Musée McCord d'histoire canadienne. Son arrivée au Musée a été précédée par une vaste correspondance entre Alice J. Turnham, directrice à l'époque des McGill University Museums, et les représentants des parties intéressées dans la transaction du dépôt de la Collection Notman au McCord, notamment 1'Associated Screen News Limited, le Maclean's Magazine, la Maxwell Cummings Family Foundation et l'Empire Universal Films Limited. ${ }^{2}$

Après la mort de William Notman en 1891, le studio "William Notman \& Son" poursuit la tradition de servir la population montréalaise tout en étant opéré par ses deux fils, William McFarlane et Charles. ${ }^{3}$ En 1935, l'entreprise familiale est vendue à l'Associated Screen News Ltd. par Charles qui, malgré son âge et mêne après cette vente, essaie de continuer le travail de son père pendant plusieurs anuées. ${ }^{4}$ Le décès de Charles Notman survenu en 1955 de même que certains changements structuraux dans l'Associated Screen News Ltd. engendrent un moment crucial pour l'avenir de la Collection Notman. La direction décide de démanteler le studio en séparant l'équipement photographique des archives contenant des centaines de milliers de négatifs en verre, de photographies et de nombreux albums de même que les répertoires de clientèle et plusieurs autres documents afin d'en faciliter la vente. ${ }^{s}$

L'équipement de la firme Notman de mêrne que les droits visant l'utilisation du nom de "William Notman \& Son" sont achetés par Niels Montclair, employé du studio. ${ }^{6}$ En ce qui concerne les négatifs et les épreuves, la situation n'est pas bien précisée dans les toutes premières lettres se rapportant au processus d'acquisition de la Collection Notman. Le 15 avril 1954, la directrice des McGill University Museums envoie une lettre à W.J. Singleton de l'Associated Screen News Ltd. Iui demandant des informations sur l'avenir des "photographies historiques du vieux Montréal". ${ }^{7}$ Selon Madame Alice J. Turnham, le dépôt à l'Université McGill des objets de la Collection Notman ne peut que rendre hommage à la famille Notman tout en enrichissant les collections du Musée McCord et servir le public, malgré le fait qu'il soit fermé depuis 1936. Pour Madame Turnham, il est essentiel de préserver tout ce qui importe à l'histoire du Canada. La réponse de Monsieur W.J. Singleton démontre qu'il y a conflit d'intérêt. Celui-ci entrevoit une autre possibilité de préservation. Il suggère le dépôt de la collection aux Archives Publiques du Canada à Ottawa car William Notman a photographié dans divers endroits autres que Montréal. ${ }^{8}$

Selon la documentation disponible anx Archives photographiques Notman, il apparaît que la décision finale a été prise à la fin de l'année 1955 puisqu'une lettre de Murray Briskin, assistant exécutif du président de l'Associated Screen News Ltd, en date du 8 décembre, confirme à Alice J. Turnham que la collection peut être cédée aux McGill University Museums pour un montant fixé à 25,000\$. De plus, il l'informe que pour alléger les coûts et faciliter ainsi la transaction, Messieurs Maxwell Cummings et Paul Nathanson feront don de 5,000\$ chacun afin d'aider l'Université McGill à acquérir Ja Collection Notman. $\mathrm{La}$ lettre comporte également des conditions préliminaires qui seront, par la suite, élaborées scrupuleusement. Le 16 décembre, dans sa téponse, la directrice des musées exprime sa satisfaction mais elle avoue son anxiété face à l'avenir de la collection photographique sachant les nombreux problèmes auxquels ont à faire face les musées de l'Université. Aussi, elle informe Murray Briskin que l'éditeur Ralpb Allen đu Maclean's Magazine a proposé sa collaboration à l'acquisition des négatifs et des épreuves pour le McCord. Pour ce faire, il se dit prêt à payer la différence du montant total, soit $15,000 \$$. Cette proposition se veut être un échange pour l'obtention des droits de préemption, le nombre d'années devant être établi sur la publication d'un nombre de photographies 


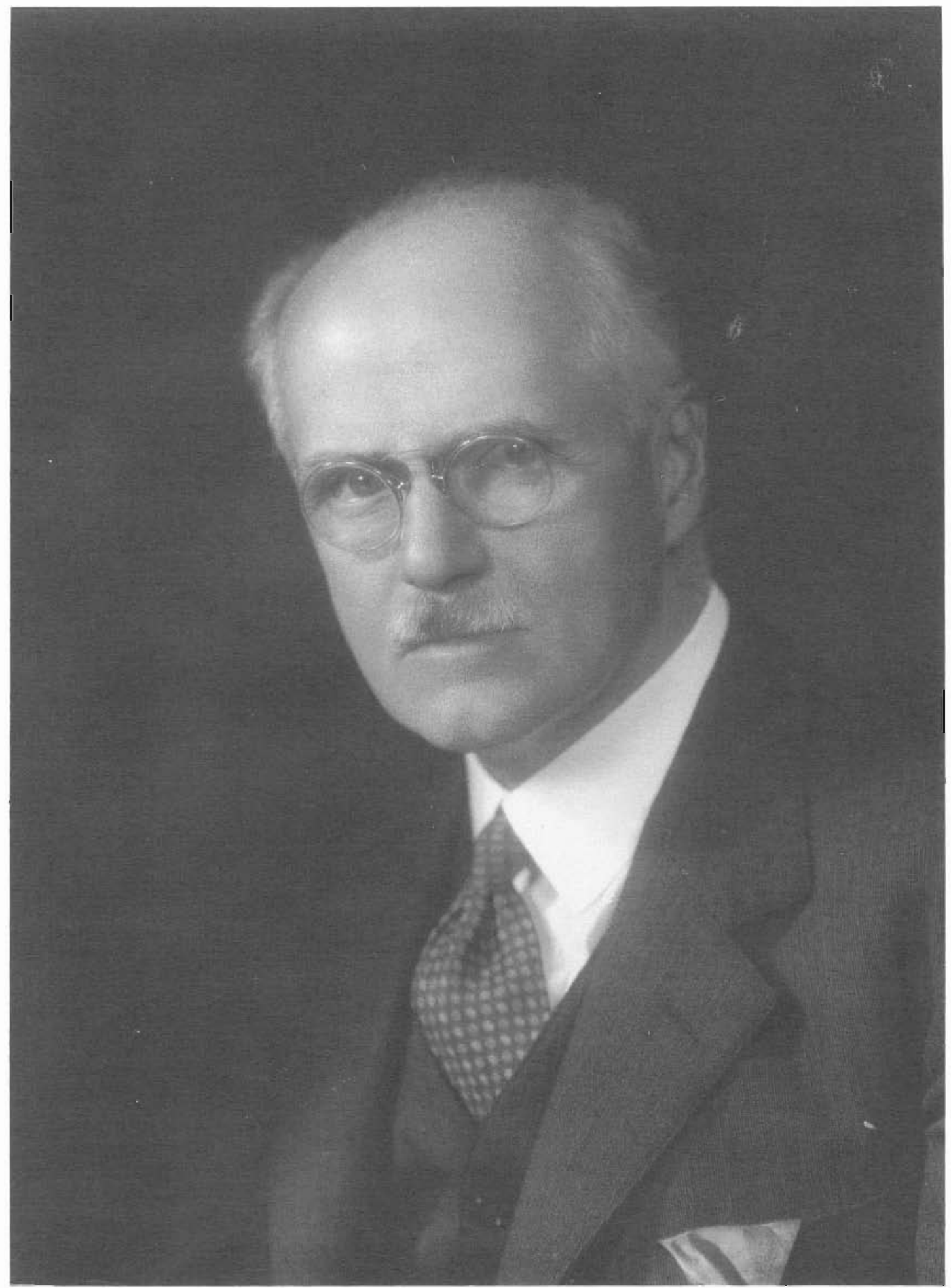

Charles Notman, ca. 1935

(Musée McCord d'histoire canadienne, archives photographiques Notman) 
provenant du Studio Notman et choisies selon les besoins du Magazine. ${ }^{9}$ A cette fin, Ralph Allen voit la nécessité d'effectuer un inventaire du contenu de la collection, le coût pouvant être partagé entre l'Université et le Maclean's. ${ }^{10}$

La correspondance effectuée entre les parties démontre le peu d'enthousiasme de la part de l'Université, voire même le peu d'intérêt, à soutenir Madame Turnham dans ses efforts de conclure l'entente. Tout semble vouloir contribuer au ralentissement du processus de règlement. La période des vacances et celle du Nouvel An s'ajoutent à la déjà lourde bureaucratie nécessaire à donner le feu vert provenant du University Museums Committee afin qu'elle procède à la dernière mise au point visant l'acquisition et la mise en valeur de la collection." $\mathrm{De}$ leur côté, la direction du Maclean's Magazine, de la Maxwell Cummings Family Foundation de même que celle de L'Empire Universal Films Limited démontrent leur volonté de simplifier la transaction à l'aide de dons additionnels servant à couvrir le montant relatif à la taxe de vente provinciale, soit $5 \% .^{12}$

L'ampleur de la correspondance concernant cette acquisition pendant les mois de mai et juin 1956 de même que leur contenu, laissent croire que le tout est conclu au cours de cette même période. En effet, en mai et en juin, les parties impliquées font l'échange de copies de propositions relatives aux conditions, de mémorandums, de lettres de remerciement, de reçus de charité et enfin, d'un projet d'offre officielle de vente de la "Notman Photographic Collection". ${ }^{13}$ Ce projet est préparé à la demande de Murray Briskin de l'Associated Screen News Ltd., par le bureau d'avocats Magee, O'Donnell \& Byers. ${ }^{14}$ Le 28 juin 1956, William Bentley écrit au Maclean's Magazine pour déterminer les conditions d'utilisation de la "Notman Photographic Collection" par le magazine et pour finalement formaliser un accord entre eux et l'Université McGill. Le troisième point de cette lettre stipule que:

For a period of approximately three (3) years expiring on August 31st, 1959, MacLean's Magazine shall, except as herein below noted, have the absolute rights to the commercial use of any and all pictures in the said Collection.
Le 6 août 1956, Alice J. Tumham fait parvenir un chèque au montant de $26,250 \$$ à Murray Briskin de 1'Associated Screen News et lui offre ses remerciements pour le soutien qu'il a su lui apporter tout au long du processus d'acquisition. La partie théorique de la mission relativement à la préservation du patrimoine est accomplie.

La collaboration entre le Maclean's Magazine et Alice J. Turnham, pour ce qui est de la collection photographique, est excellente. D'après la documentation des Archives photographiques Notman, on peut affirmer que cette transaction s'est révélée des plus positives car elle a permis de sortir la collection de l'ombre. Les éditeurs du magazine Maclean's, de par leurs exigences, ont donné une renaissance à la renommée de l'oeuvre de William Notman et de celle de son studio.

LA VIE MUSÉALE ET PUBLIQUE DE LA "COLLECTION NOTMAN"; LA DIVERSITÉ DES PROBLÈMES PAR RAPPORT À SON UTILITÉ

La précarité financière alliée au manque d'espace et de personnel qualifié pour faire fonctionner les musées de I'Université McGill de façon appropriée et intelligente en fonction de leur vocation ont aussi affecté dans une certaine mesure les activités relatives à la Collection Notman. Dans son allocution lors du lancement qui a lieu au Musée Redpath, le 9 novembre 1956, Madame Turnham suggère que la nouvelle acquisition soit l'objet d'une série de traitements spécialisés qui rendront la collection inaccessible pour longtemps. ${ }^{15}$ Elle exprime également son inquiétude au sujet de l'entreposage des épreuves et des négatifs car ceux-ci, bien qu'ils soient destinés au Musée McCord, seront temporairement logés à la Bibliothèque Redpath.

Comme il a été mentionné plus haut, la coopération est à son meilleur entre le Maclean's Magazine et Alice J. Turnham de même qu'avec le personnel qui travaille au fil des années sur la collection photographique. Au départ, l'Université a dû accorder des moyens à la directrice pour effectuer l'inventaire préliminaire. Cette forme particulière de recherche, déteminée par les besoins publicitaires du Maclean's, a un impact immédiat sur la diffusion du contenu de la collection. Plusieurs articles publiés dans le populaire magazine 


\section{Les Archives photographiques Notman du Musée McCord}

provoquent l'intérêt du public pour la collection. La "découverte" des photographies du Studio Notman inspire un certain nombre de canadiens à chercher leurs origines. ${ }^{16}$ Bientôt, les autres média manifestent leur volonté d'utiliser le phénomène qu'est devenu l'oeuvre Notman, dans leurs productions. ${ }^{17}$ Suite à cet intérêt public, Alice J. Turnham donne au moins trois conférences sur la Collection Notman, deux en automne 1957 et une en $1960 .^{18}$ Mais avant, elle élabore une exposition dédiée à William Notman et intitulée !!CAMERA!! au cours de l'été 1957. Cette exposition, présentée du 15 mai au 15 septembre, est basée sur une sélection de photographies de la nouvelle acquisition, qui sont elles-mêmes accompagnées d'objets de l'époque Victorienne provenant des collections du Musée McCord. ${ }^{19}$ Il est nécessaire ici de mentionner deux autres sources de soutien concernant la mise sur pied de cette exposition. Pour l'occasion, quelques documents et albums de même qu'une partie de l'équipement provenant de l'ancien Studio Notman ont été prêtés à Alice $\mathbf{J}$. Turnham par leur propriétaire, Niels Montclair. ${ }^{20}$ Pour sa part, l'éditeur général du Maclean's Magazine, Pierre Berton, a une fois de plus fourni un certain nombre d'agrandissements de photographies provenant du Studio. ${ }^{21}$ L'exposition organisée pour les raisons que l'on connaît au Musée Redpath est ouverte au public tous les jours, sauf le dimanche, de $9 \mathrm{H} 00$ à $17 \mathrm{H} 00$ et l'entrée est gratuite. ${ }^{22}$

\section{QUESTION D'ENTREPOSAGE}

L'acquisition d'une collection aussi volumineuse dont les problématiques s'avèrent particulièrement complexes, relève de l'héroïsme quand on considére la situation dans laquelle se trouvaient les musées de l'Université McGill à l'époque. Directrice des McGill University Museums depuis 1951, Alice J. Turnham voit cette opportunité comme un autre défi à relever, comme une continuité de l'oeuvre de E. Lionel Judah, son professeur de muséologie. ${ }^{23}$

Les préparatifs en vue du déménagement de la Collection Notman du sous-sol de l'édifice de l'Associated Screen News Ltd. à la Bibliothèque Redpath débutent le 20 janvier 1956, c'est-à- dire avant l'accomplissement du processus formel d'acquisition. En effet, dans une lettre datée ce même jour, Alice J. Turnham décrit son plan stratégique au directeur de
l'Université, F. Cyril James. Ce plan, établi lors de la réunion du University Museums Committee le 16 janvier, prévoit que face au manque d'espace approprié pour placer la collection entière au même endroit (Musée McCord), l'alternative la plus favorable est la "Lincoln Room" à la Bibliothèque Redpath. Madame Turnham mentionne également que si le directeur est d'accord, l'opération du déménagement et le travail du professeur John Cooper qui doit débuter l'inventaire de la collection, peuvent être entrepris immédiatement. Cette lettre est intéressante car pour une première fois et il y en aura plusieurs autres, la directrice des McGill University Museums suggère une solution au problème d'entreposage. Elle propose tout simplement de jeter une partie considérable des négatifs en verre. ${ }^{24}$ Dans un document intitulé Notman Photographic Collection, 1856-1945, Now Housed at Associated Screen News, 2000 Northcliffe Ave., N.D.G., elle maintient son opinion et veut l'appliquer à l'ensemble de la collection. Selon une note manuscrite, ce document est parvenu les 9 et 10 février 1956 à Messieurs Murray Briskin de l'Associated Screen News Ltd. et Ralph Allen du Maclean's Magazine. Le point numéro 5 du document se lit comme suit:

That once Maclean's list [is] completed, the University shall have the right to discard any portions of the remainder of the collection, which it may consider irrelevant to its own needs; the same right to apply to all pictures on Maclean's list, once Maclean's prior right has expired;

Cette approche dogmatique est également vue par Madame Turnham comme une solution applicable aux albums. Les alternatives particulières proposées par la directrice sont notées dans les "Recommandations" du "Progress Report" et envoyées aux parties impliquées le 26 avril 1956. Dans ce rapport, elle souligne que les albums doivent être démantelés et les photographies placées dans un dossier "for ready reference", mais elle ajoute que cette méthode peut exiger "a great deal of labour". Elle propose donc une autre procédure, notamment d'effectuer des photostats de chacune des pages de tous les volumes et de couper les photographies individuellement pour les placer ensuite dans un dossier. "The uriginal volumes could then be kept if that seemed advisable or could be eliminated." Heureusement, il semble que rien n'ait été éliminé selon 
Les Archives photographiques Notman du Musée McCord

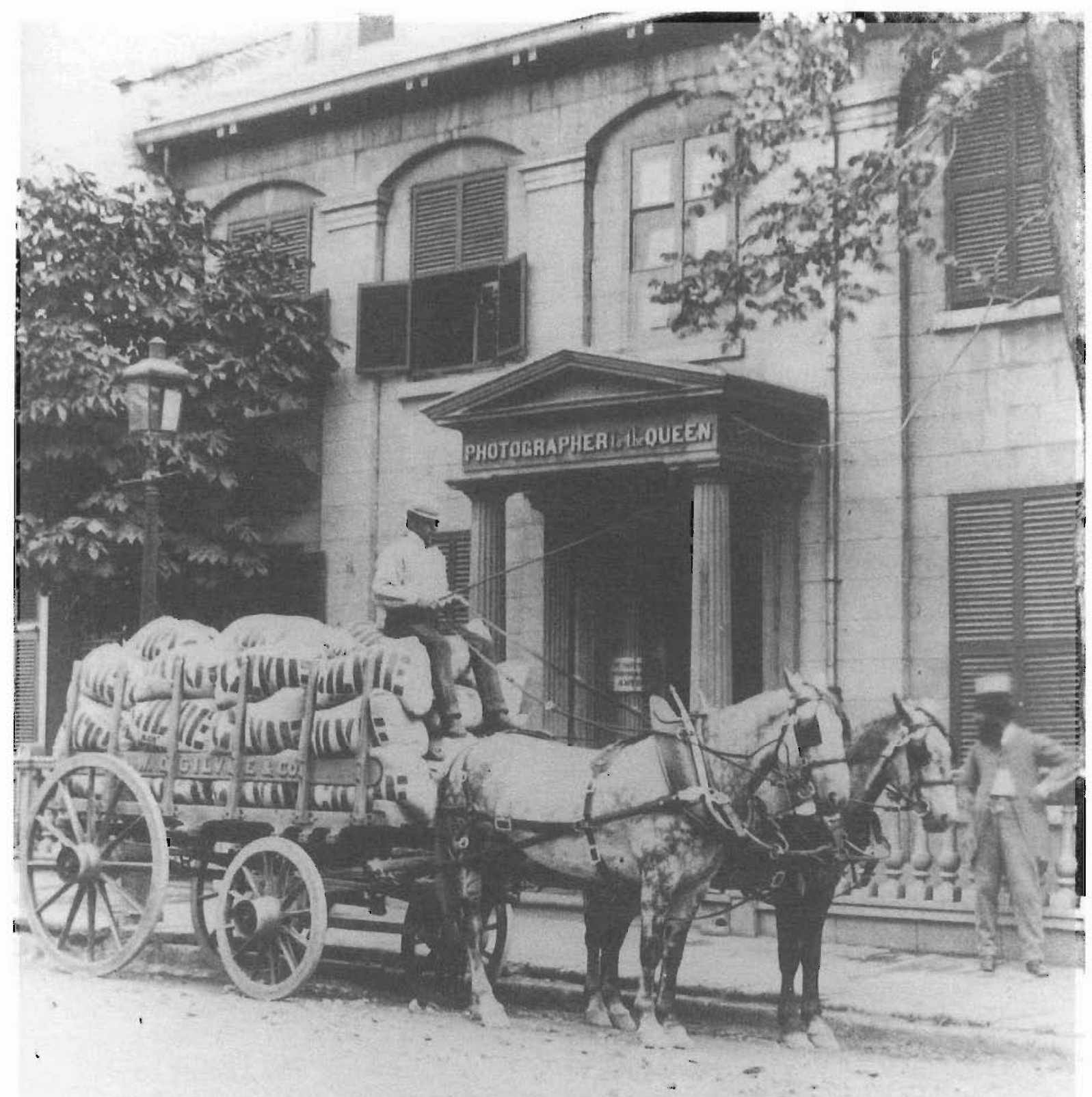

Studio Notman sur la rue Bleury, Montréal, avec la charrette de W. Ogilvie et Cie, 1864 (Musée McCord d'histoire Canadienne, Archives photographiques Notman) 
Les Archives photographiques Notman du Musée McCord

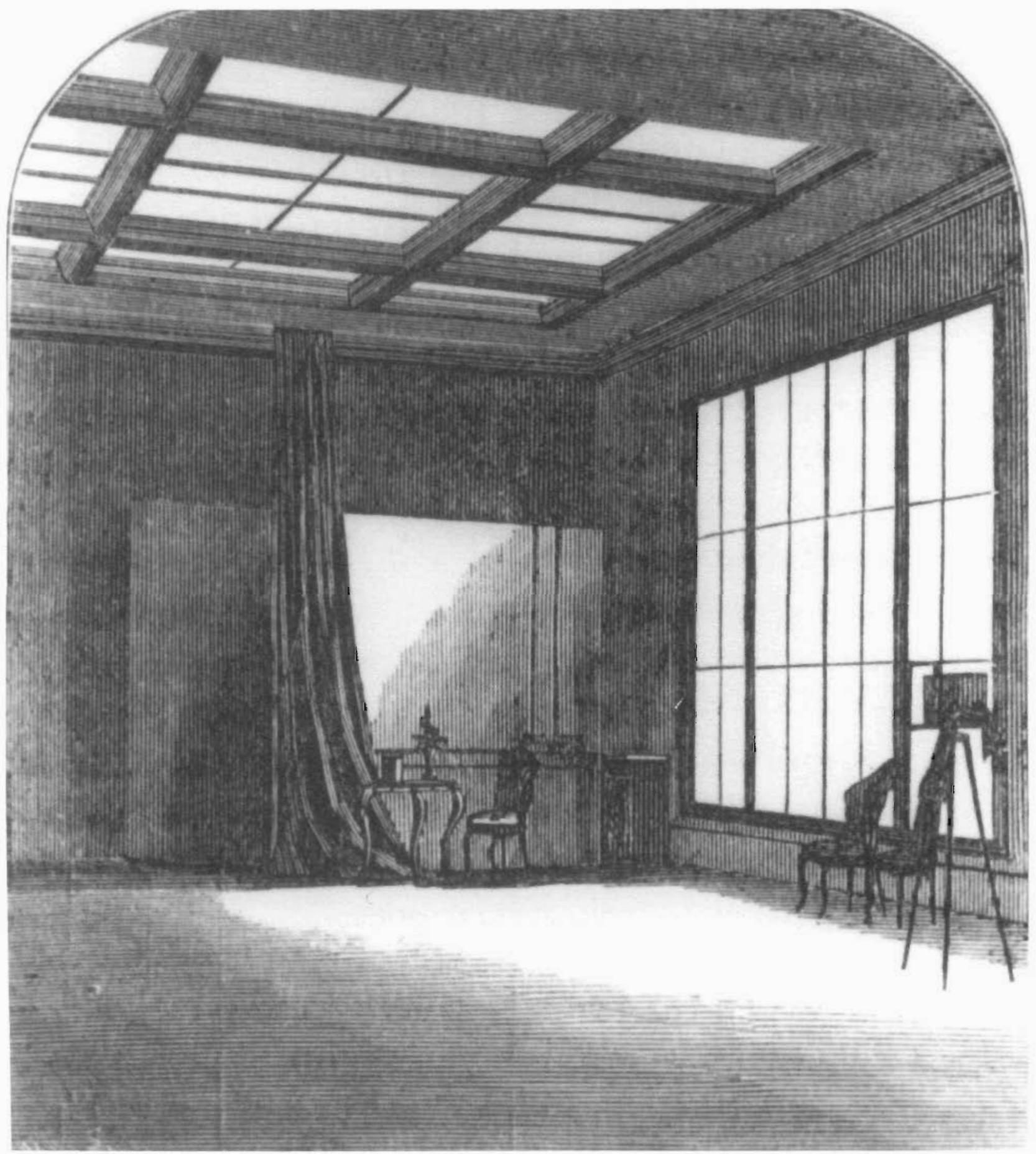

Studio "A" de Notman. Philadelphia Photographer, August 1866. (Musée McCord d'histoire Canadienne, Archives Photographiques Notman) 


\section{Les Archives photographiques Notman du Musée McCord}

les méthodes suggérées par Madame Turnham sauf les négatifs en nitrate endommagés par leur autodestruction et par les conditions d'entreposage. ${ }^{25}$

La réception des informations révélant l'ampleur réelle de la collection de même que la description des espaces de rangement posent un nouveau problème à Alice J. Turnham qui en avise le directeur, F. Cyril James: "The weight of 500,000 glass slides would be too great for the floor of the Lincoln Room". ${ }^{26}$ Elle suggère d'emblée un autre espace dans le même édifice. Le directeur étant favorable à la proposition, on pense immédiatement au transport mais les démarches sont arrêtées. Bien que la documentation semble incomplète sur le sujet, quelques lettres échangées au cours du mois de mars 1957 mentionnent que les négatifs sont affectés par les mauvaises conditions d'entreposage car on a découvert la présence de petites bêtes connues sous le nom de poisson d'argent. La lettre de Alice J. Turnham à Murray Briskin datée du 9 février 1956 donne à penser que la mauvaise condition des négatifs était déjà connue car Madame Turnham propose que: "The glass plates could be transferred later, as they are part of yet another operation".

The Pestroy Company Limited est choisie pour effectuer un test de désinfection par fumigation sur une partie des négatifs avant de procéder au traitement de l'ensemble de ceux-ci. Le test n'a démontré aucun dommage visible sur l'émulsion photographique des négatifs, ce qui n'exclut aucunement l'apparition de changements à long terme, comme le signale George Rance de l'Associated Screen News Ltd. ${ }^{27}$

La fumigation de la totalité des négatifs a lieu les 23 et 24 mars 1957 et dès le 25 , la compagnie Morgan Storage and Van Lines Ltd. entreprend la préparation des négatifs en verre pour les déménager à la Bibliothèque Redpath. ${ }^{28}$ Cette opération se termine avant le 16 avril 1957. Contrairement aux prévisions antérieures du nombre de négatifs en verre $(500,000)$, on n'en dénombre que 300,000 après le déménagement. ${ }^{29}$

\section{LE CATALOGAGE INITIAL}

La vérification des albums et des répertoires de la clientèle débute un an avant le transfert des négatifs.
Au cours du printemps 1956, le professeur John Cooper du département d'histoire de l'Université McGill examine le contenu en marquant les photographies à l'aide d'insertions en couleur selon des thèmes. ${ }^{30} \mathrm{Ce}$ système de classification thématique des photographies, établi à la demande du Maclean's Magazine, est toujours utilisé aux Archives Photographiques Notman. Le professeur Cooper ne travaille que 75 heures sur la collection. Il est accompagné par le Dr. Gerhard R. Lomer, ancien bibliothécaire de l'Université McGill, qui est employé pour effectuer un inventaire détaillé des albums et superviser le catalogage des photographies en prévision de la finalisation de l'acquisition et de la sélection d'un nouveau catalogueur. ${ }^{31}$

Il s'avère très difficile de définir la fluctuation du personnel employé pour travailler sur la collection des négatifs et des épreuves provenant du Studio Notman et déposés à la bibliothèque Redpath. La documentation disponible aux Archives photographiques du Musée McCord parait peu complète mais on peut $y$ apprendre que dès l'hiver 1956, Anne E. McKim est chargée du catalogage. ${ }^{32}$ La préparation des négatifs pour leur transfert du sous-sol de l'Associated Screen News Ltd. a été supervisée, du côté de l'Université, par Margaret Lukis Lambert. Cette dernière, une amie proche de la famille Notman, a travaillé auparavant au Studio avec Charles Notman à titre de photographe. Étant donné sa connaissance de la collection, il est certain que son aide est pertinente. ${ }^{33}$ En 1957, on procède à l'engagement de deux autres personnes pour continuer le traitement de la collection, notamment John A. Kennerley et Barbara M. Chadwick. Madame Chadwick débute comme assistante catalogueur et y travaille jusqu'en 1960. Elle fait aussi le classement des épreuves et des négatifs et répond aussi aux nombreuses demandes de la clientèle. Les deux dernières années, elle est responsable de la collection. ${ }^{34} \mathrm{Au}$ cours de cette période, pour cause de maladie, Madame Chadwick est remplacée temporairement par Margaret Lukis Lambert. ${ }^{35}$

\section{QUESTION DE CONSERVATION}

Les années suivant l'acquisition sont marquées par la recherche concernant la préservation. L'état de conservation des négatifs et des épreuves ne permet pas une utilisation illimitée. Pour assurer une manutention 
Les Archives photographiques Notman du Musée McCord

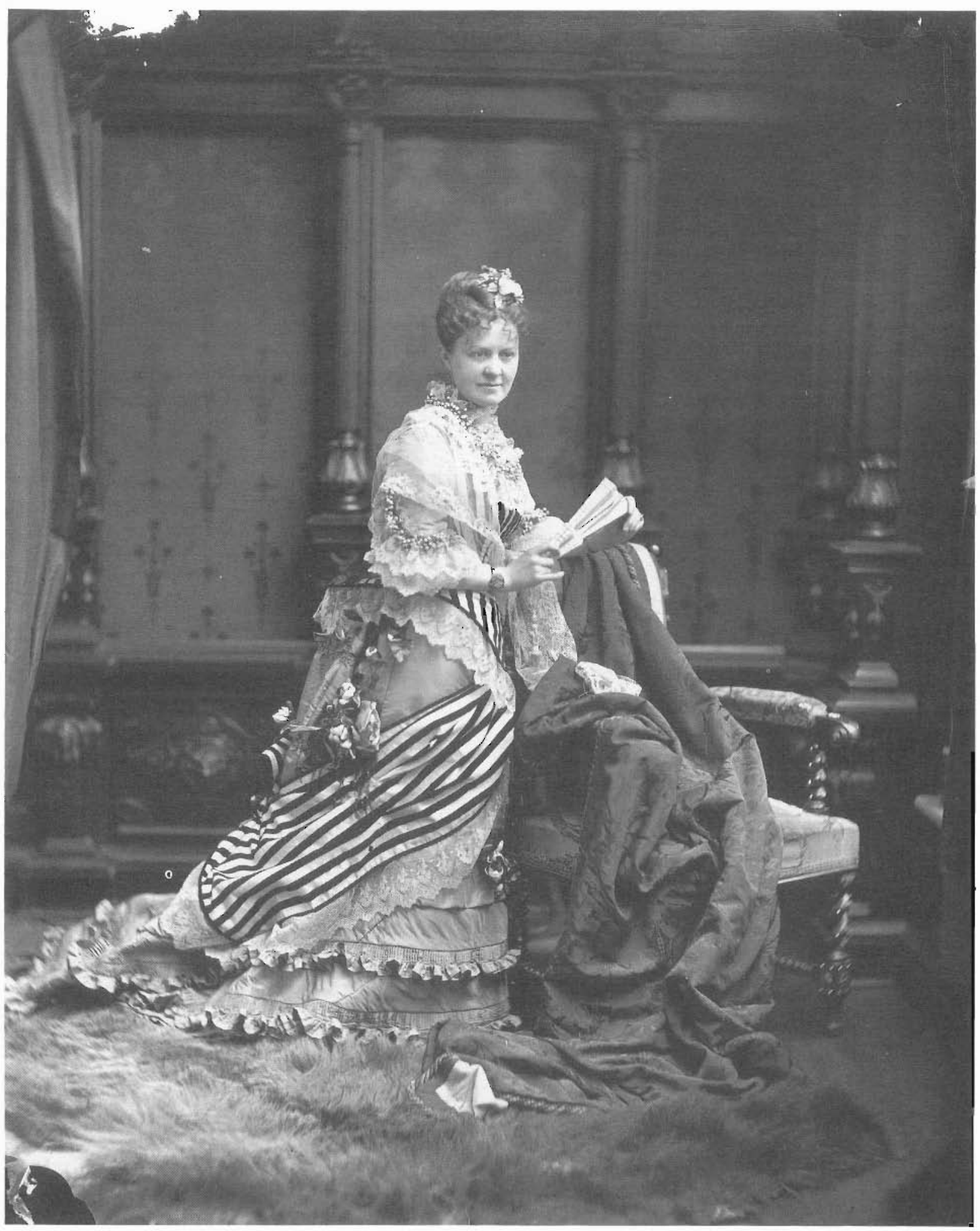

Mme W.B. Lindsay, 1876. (Musée McCord d'histoire Canadienne, Archives photographiques Notman) 
Les Archives photographiques Notman du Musée McCord

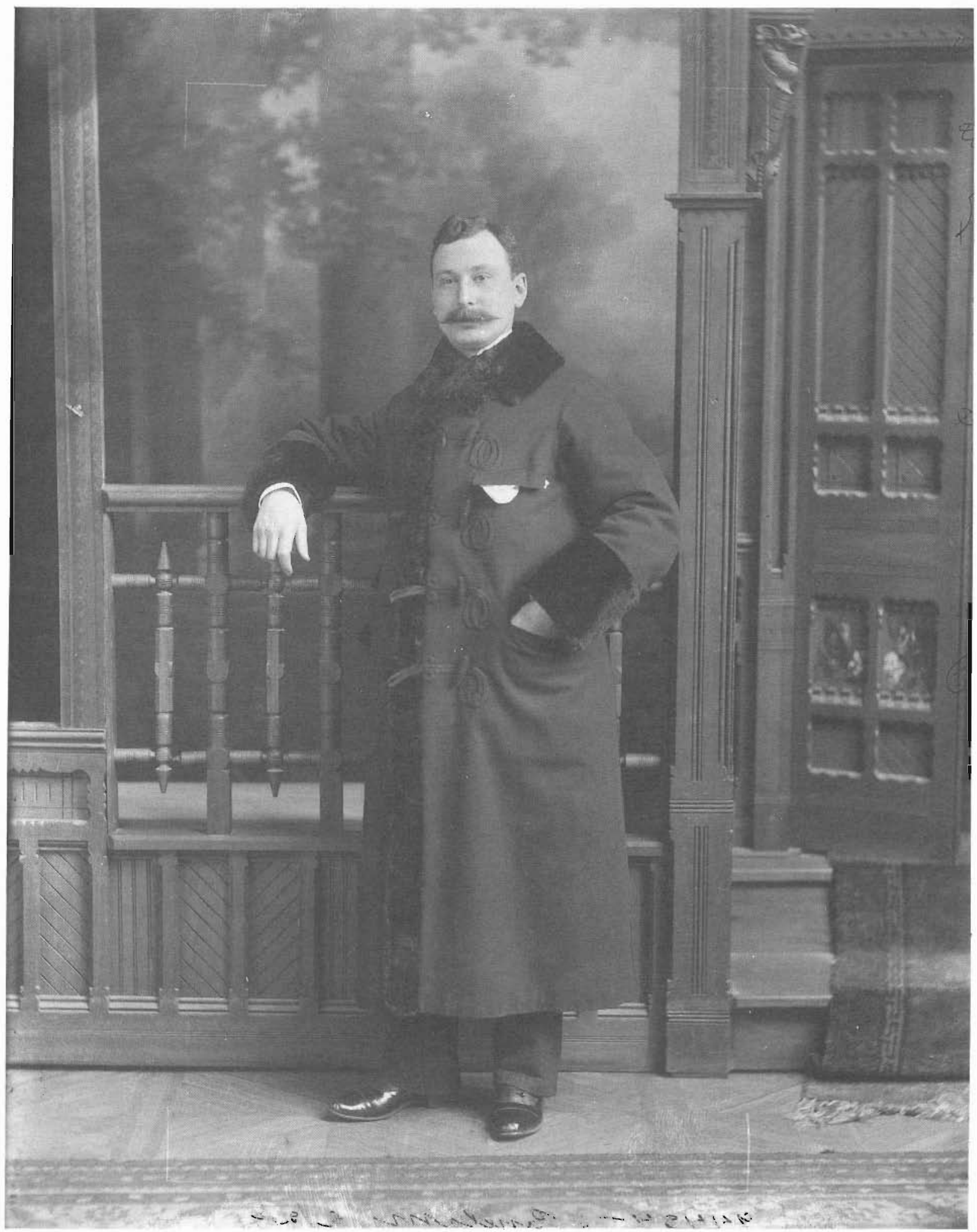

Mortimer Davis, 1891. (Musée McCord d'histoire Canadienne, Archives photographiques Notman) 
qui soit sécuritaire, il faut trouver et élaborer certaines mesures de protection des objets. La situation se voit aggravée par le manque de service photographique professionnel attaché à la collection. ${ }^{36}$ Selon l'entente conclue entre l'Université McGill et le Maclean's Magazine pour l'acquisition de la collection, le contenu aurait dû être accessible le plus rapidement possible. Pendant trois années les négatifs en verre et les épreuves originales choisis pour être publiés ont été envoyés à plusieurs reprises à Toronto. Soulignons que les assurances tous risques sur la durée de leur séjour aux locaux du magazine sont payées par l'Université McGill. ${ }^{37}$

Pour faire face à un nombre croissant de demandes provenant du public, des institutions professionnelles et commerciales, pour l'utilisation des photographies du Studio Notman, on fait appel à la firme Arnott Rodgers de Montréal afin qu'elle effectue des épreuves et des copies de négatifs. ${ }^{38}$ Mais une fois encore les objets originaux sont soumis à des déplacements répétés ce qui contrevient, particulièrement dans le cas d'objets fragiles comme les négatifs en verre, aux règles de sécurité et surtout de préservation.

Le catalogage des photographies soulève un questionnement en regard de la méthode de préservation de cette partie de la collection par rapport à la nécessité de son utilisation. La recherche sur ce problème commence à la fin de 1956. Le sujet de la préoccupation sont les pochettes utilisées pour la conservation des épreuves, soit la sécurité de leur utilisation car celles-ci sont faites en acétate de cellulose. De plus, leur coût est considérablement élevé. Après avoir pris connaissance des expertises déjà établies, comme celle de la Canadian Chemical Co. Ltd., on demande au gouvernement du Canada de faire des tests additionnels concernant la sécurité du matériel par rapport aux objets muséaux, plus particulièrement aux photographies. Suite aux résultats, les pochettes sont acceptées comme un moyen permettant une manipulation sécuritaire des épreuves. ${ }^{39}$

Les albums font aussi l'objet d'une recherche concernant leur état de conservation de même que la possibilité de les rendre accessibles aux chercheurs. Le démantèlement, appliqué déjà sur vingt-six albums, est momentanément abandonné. Notons que ce sont tous des albums de photographies mesurant 5 pouces sur $7 .^{40}$ Au cours de l'année 1959, les personnes impliquées dans la Collection Notman font la "découverte" du laminage qui, en étant déjà utilisé pour préserver les documents, pourrait être appliqué sur les albums pour éviter leur destruction. ${ }^{41}$ Les résultats de la recherche sur le laminage, poursuivie en hiver 1959 par Margaret Lukis Lambert et Alice J. Turnham, sont soumis le 28 octobre de cette même année au University Museums Committee. La décision de ce dernier est de rejeter la méthode du laminage et de continuer le démantèlement des albums, d'insérer les photographies dans une pochette en acétate et enfin, de les classer verticalement dans un cabinet en métal. ${ }^{42}$ Selon la directrice des musées de l'Université McGill, cette méthode est recommandée dans le cours "Principles of Archival Management" de l'Université Harvard et acceptée aux Archives Publiques du Canada et la Library of Congress à Washington; elle est également utilisée par la majorité des grands musées. ${ }^{43}$

In order to demonstrate the manner in which $\mathrm{Mr}$ Notman originally kept his prints, it might be well to retain one of the less important volumes as an exhibition specimen which eventually could be laminated. ${ }^{44}$

La décision du University Museums Committee d'abandonner le "projet laminage" a été prise en regard de l'aspect financier et non dans un esprit de préservation des objets. Nul n'a songé à cette époque que toute intervention sur des objets du patrimoine, placés dans un musée, doit être minimisée et surtout être réversible. Ainsi donc, la méthode du laminage de même que celle du démantèlement des albums sont inquiétantes car elles ne sont évidemment pas réversibles.

\section{LES ARCHIVES PHOTOGRAPHIQUES NOTMAN}

Suite à la décision d'unifier la Collection Notman à une autre déjà établie dans les archives du Musée McCord par son fondateur, David Ross McCord, l'appellation se voit changée. Dès le début des années 60 , il est question maintenant des Archives photographiques Notman car la majorité du contenu provient de l'ancien Studio Notman. ${ }^{45}$ 
Les Archives photographiques Notman du Musée McCord

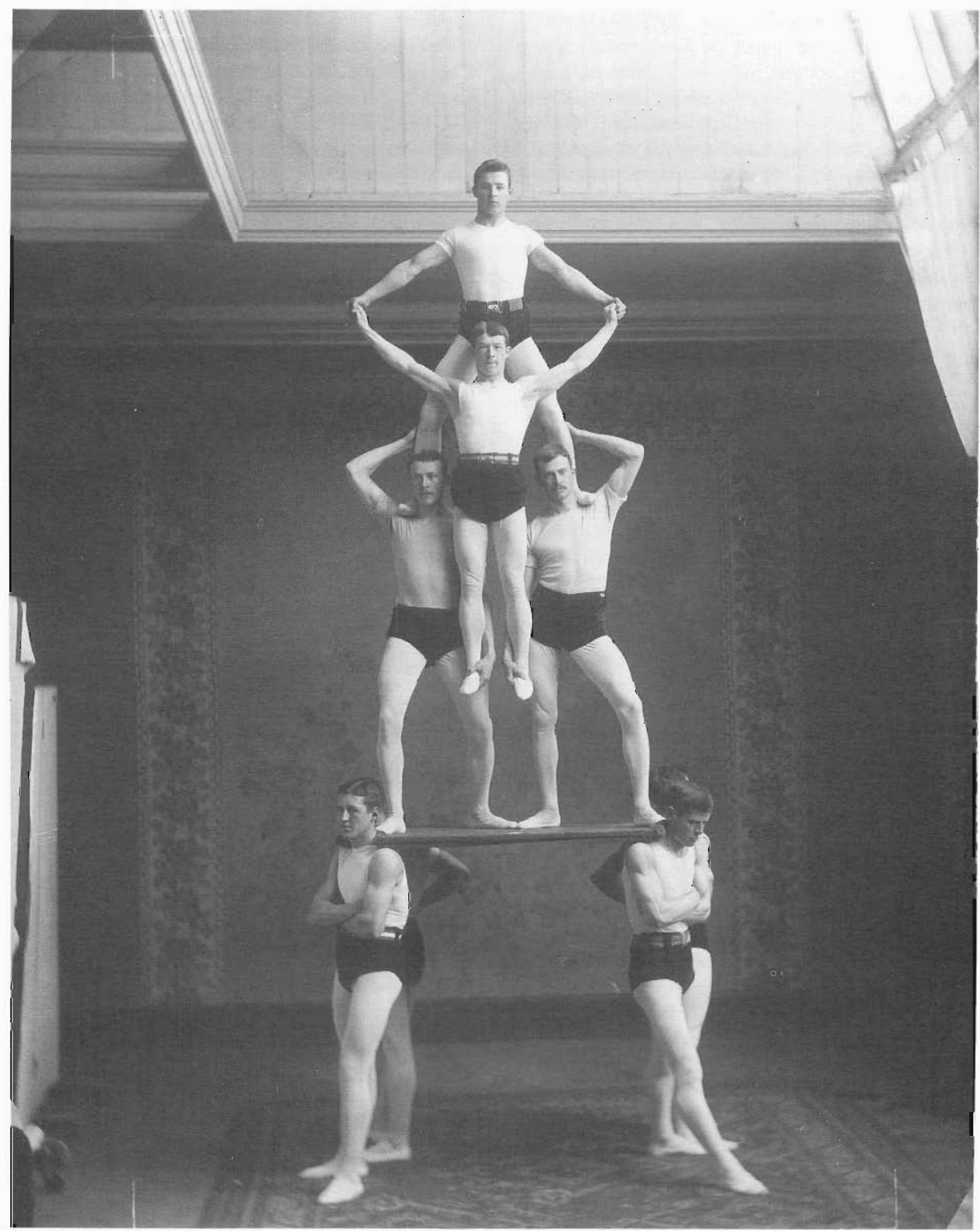

Groupe de gymnastes, 1891. (Musée McCord d'histoire Canadienne, Archives photographiques Notman) 


\section{Les Archives photographiques Notman du Musée McCord}

En 1965, les autorités des McGill University Museums ouvrent un poste de conservateur de la photographie pour les Archives photographiques Notman du Musée McCord. Celui-ci est comblé par Stanley G. Triggs, photographe professionnel. Dès son arrivée, Monsieur Triggs entreprend une vérification du système de catalogage, du classement, du service à la clientèle et de la préservation des objets de même que du nombre d'employés en regard des autres collections photographiques d'importance au pays. ${ }^{46}$ Parallèlement, il met sur pied une chambre noire afin de répondre aux besoins du Musée McCord relativement aux expositions basées sur la collection photographique ou encore, pour répondre aux demandes provenant du public et ceci, sans déplacer les négatifs historiques en dehors des Archives. ${ }^{47}$ La période d'improvisation pour cette collection est terminée.

L'approche professionnelle du conservateur, renforcée par une forte passion pour l'oeuvre de William Notman, donne bientôt des résultats visibles en ce qui a trait au travail exécuté par le personnel des Archives photographiques. En 1967, un livre intitulé Portrait of a Period, a Collection of Notman Photographs 1856 to 1915 est préparé et édité par J. Russell Harper et Stanley G. Triggs. Au cours de la même année, une exposition itinérante de photographies provenant du Studio Notman est envoyée à travers le Canada. ${ }^{48}$

En mars 1968, la Collection Notman est l'objet de la planification d'un déménagement, cette fois-ci, de la Bibliothèque Redpath au Musée McCord. On prévoit l'emballage du 13 au 17 mai et le transfert pour la semaine suivante. ${ }^{49}$ Pour ce qui est du bureau, son déménagement vient longtemps après, soit en janvier 1969. Pour cette raison, les portes des Archives photographiques Notman sont fermées entre le 20 décembre 1968 et le 20 janvier de l'année suivante. ${ }^{50}$ Ce déménagement n'est pas exclusif aux collections photographiques du McCord, il s'étend également à toutes les collections du Musée, entreposées temporairement à la Maison Hodgson.

C'est un moment important dans l'histoire des Archives photographiques Notman de même que dans celle du Musée McCord qui, au printemps 1971, ouvre ses portes au public après avoir été fermé pendant 35 ans. Il est à noter que cette réouverture est rendue possible grâce aux efforts de Madame Isabel Barclay Dobell, conservatrice en chef du McCord à l'époque. Connaissant la situation désastreuse des collections du musée, y compris les Archives photographiques, Madame Dobell demande l'appui de Walter Stewart, mécène bien connu de la culture, et enfin sollicite l'aide financière de la McConsell Foundation. ${ }^{51}$ L'adaptation du bâtiment du Centre social des étudiants de l'Université McGill, construit entre les années 19041906 par Percy Erskine Nobbs, pour les besoins du musée est confiée à l'architecte Guy Desbarats et au dessinateur Clifford Williamson. ${ }^{52}$ Lorsque cette phase est terminée et que les collections trouvent leur place au sein du nouveau site, le Musée McCord propose au public montréalais une grande exposition, préparée par les Archives photographiques Notman. Pour l'occasion, on emprunte le titre "Portrait of a Period" du livre publié en 1967. C'est une première exposition majeure des photographies effectuées dans le Studio Notman entre 1856 et 1880 (époque du "wet-plate" dans l'histoire de la photographie). La différence entre le livre et l'exposition réside dans le fait que toutes les photographies tirées des négatifs originaux et choisies méticuleusement par le conservateur, Stanley Triggs, et arrangées par le dessinateur Clifford Williamason, n'ont jamais été publiées ou vues publiquement avant cette exposition. Tout comme le musée à ce moment, l'exposition est ouverte au public seulement trois jours par semaine, de $11 \mathrm{H} 00$ à $18 \mathrm{HOO}$ pendant six mois. ${ }^{53}$ Le succès de cette exposition a peut-être influencé la décision du Conseil des Arts du Canada d'accorder au Musée une subvention de l'ordre de 5,000\$ en septembre 1971, qui sera versée le premier novembre 1972 avec la stipulation suivante: "To help catalogue and preserve the Notman photographic collection". .4

\section{LE CONTENU}

Les Archives photographiques Notman du Musée McCord contiennent plusieurs collections photographiques. La Collection Notman est la plus grande et la plus homogène, puisqu'elle s'est enrichie, en juin 1958, des dons effectués par Niels Montclair et George Dudkoff. Monsieur Montclair, propriétaire de l'équipement photographique de l'ancien Studio Notman, cède avec empressement les objets à l'Université McGill comme le lui suggère la directrice des musées, Alice J.Turnham. ${ }^{55}$ Les autres collections 


\section{Les Archives photographiques Notman du Musée McCord}

importantes incluent les négatifs et les épreuves de Alexander Henderson, William Hanson Boorne, Peter Pitseolak et Charles Millar. De façon générale, on retrouve dans ces archives toute I'histoire de la photographie. Celle-ci est racontée à l'aide de plus de 100 daguerréotypes, ambrotypes et tintypes effectués dès 1845 , également 500 diapositives des années 1870 et 1910 , colorées à la main, sans oublier les réalisations de William Notman, aussi des équipements photographiques de l'ancienne époque jusq̨u'à nos jours, car il y a également des exemples de photographies contemporaines représentées entre autre par les travaux. de Clara Gutsche, Gabor Szilasi et Thaddeus Holownia. ${ }^{56}$ Certaines collections sont titrées du nom de leur donateurs, comme par exemple la collection Fritz Arnold, la collection Roper ou celle de Preston. ${ }^{57}$

Le nombre exact des objets se trouvant aux Archives photographiques paraît difficile à déterminer, voire même impossible. Selon Nora Hague qui travaille aux Archives depuis 1969, et qui occupe actuellement le poste d'assistante du conservateur de la photographie, l'ensemble des collections peut se chiffrer aux environs de 750,000 objets mais ce nombre pourrait être plus élevé car le processus du catalogage suit son cours. Depuis le 25 mars 1991, le Musée McCord entame son projet d'informatisation des collections québécoises. ${ }^{58}$ Les collections photographiques sont donc aussi soumises au nouveau système de catalogage. L'enthousiasme de Madame Hague qui a commencé le travail chez elle bien avant l'introduction officielle du système, a considérablement avancé le catalogage. ${ }^{59}$

Pour ce qui est de la Collection Notman, le système privilégié par William Notman lui-même facilite l'utilisation de la collection. Il est d'ailleurs encore utilisé aujourd'hui par les Archives photographiques. L'informatisation est donc concentrée sur les autres collections encore difficiles d'accès. Afin de donner une image de la situation actuelle, soulignons que 5,045 objets de la Collection Notman et 13,818 objets provenant d'autres collections sont déjà catalogués à l'aide de l'ordinateur. Ce qui totalise 18,863 objets traités. Nora Hague est soutenue dans ce travail par Heather $\mathrm{McNabb}$, assistante recherchiste. Cette équipe se voit complétée par le photographe Tom Humphry. Une personne continue bénévolement le catalogage traditionnel; mais celui-ci est loin d'être terminé. ${ }^{\infty}$

\section{CONCLUSION}

Le Musée McCord est de retour après avoir déplacé temporairement ses collections pendant trois ans à cause de son projet d'agrandissement. Malgré ce déplacement, les Archives photographiques Notman n'ont pas cessé de répondre aux demandes du public sauf pendant la période du déménagement. Il est à souligner que les collections photographiques du Musée McCord représentent une source infinie d'informations. Les chercheurs professionnels et les étudiants de toutes disciplines de même que le public en général, peuvent explorer le passé du Canada à travers le matériel historique rassemblé par les nombreuses personnes impliquées dans la création des Archives photographiques Notman, qui avaient cet intérêt commun. Même si les premières décisions concernant la méthodologie de conservation de la Collection Notman peuvent paraître aujourd'hui controversées, il nous faut comprendre les circonstances. L'histoire des Archives photographiques Notman n'est que le miroir reflétant la situation de tous les musées de l'Université McGill. La centralisation de la gestion des musées et les difficultés financières perpétuelles ont déterminé l'évolution des collections. Pendant les premières années, c'est-à dire jusqu'en 1965, les Archives n'ont pas retenu les services d'un photographe. ${ }^{61}$ Jusqu'à ce moment-là, toutes les interventions ont été initiées par une seule et même personne, notamment Alice J. Turnham. Il est évident que cette dernière a dû relever un défi majeur - sauver la collection. On peut dire, qu'à sa façon, elle y est parvenu.

En terminant, un mot sur le fonctionnement des Archives photographiques du McCord. Le rôle visant l'utilité sociale dans cette section du Musée est évident. En fait, effectuer sa propre recherche aux Archives est très satisfaisant. L'accessibilité et l'appui professionnel du personnel se traduisent par des chiffres éloquents par rapport aux autres collections. En effet, la moyenne des visiteurs est de 900 à 1000 personnes par année, ce qui inclut les visites répétées. ${ }^{62}$ Les gens viennent de tout le Canada mais aussi de l'étranger. Les activités aux Archives sont sans aucun doute parallèles aux fonctions primordiales des musées en général. Le récent agrandissement du Musée devrait donner plus d'espace pour les collections et les chercheurs. Si on ajoutait quelques membres de plus au personnel, le service et l'accès en seraient grandement améliorés. 
Les Archives photographiques Notman du Musée McCord

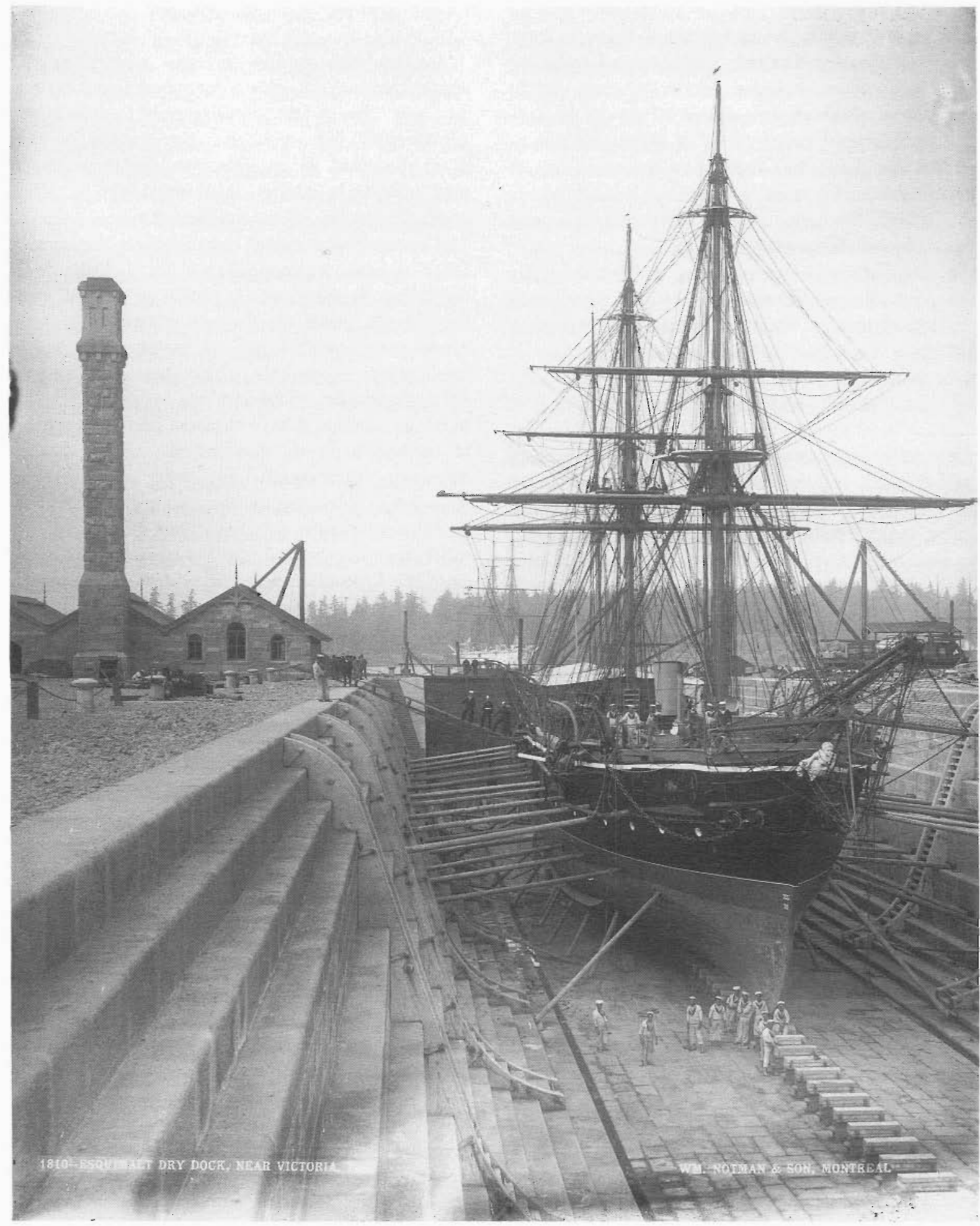

La cale sèche, Esquimalt, Columbie Britannique, 1887. (Musée McCord d'histoire Canadienne, Archives, photographiques Notman) 


\section{POST-SCRIPTUM}

Le texte ci-haut, écrit en décembre 1991, est le résultat d'une recherche effectuée au cours de l'automne de la même année. A la fin de juin 1993, le conservateur Stanley Triggs a pris officiellement sa retraite,

\section{Notes}

1. Stanley G. Triggs, William Notman. L'empreinte d'un studio, Musée des Beaux-Arts de l'Ontario, Toronto, 1986, pp. 23-26; 28-31 aussi Portrait of $a$ Period, édité par J. Russel Harper et Stanley Triggs, Montréal, McGill University Press, 1967 (texte non paginé); Glen Warner, Building a print collection. A guide to buying original prints and photographs, Van Nostrand Reinhold Ltd. , Toronto-New York-CincinnatiLondon-Melbourne, 1981, pp. 16-17; Peinture et photographie canadiennes, 1860-1900, Musée McCord, catalogue d'exposition, 1979, pp. 23-26, 29, 46; William Welling, Photography in America. The Formative years 1839-1900, New York, 1983, p. 254; Naomi Rosenblum, A world history of photography, New York, 1984, pp. 137, 236; Helmut Gernsheim, Alison Gernsheim, The history of photography from the camera obscura to beginning of the modern era, New York-St-Louis-San Francisco, 1969, pp. 299-300; Dennis Reid, Our Own Country Canada, Being an Account of the National Aspirations of the Principal Landscape Artists in Montreal and Toronto 1860-1890, National Gallery of Canada, 1979, pp. 53, 131; Michel Lessard, Les Livernois, photographes, Musée du Québec, 1987, les chapitres: "La division du travail", "Le portrait classique en buste, 1870-1914"; Larry Raskin, "William Notman, Through the looking g]ass", Dossier dans une revue intitulée Montréal, septembre 1989, p. 30; George Zimbel, "Rediscovering a Canadian pioneer", Photo District News, May 1985 pp. 54, 56; Véronique Robert, "L'album de famille du Canada", L'Actualité, octobre 1989, p. 18.

2. La documentation se trouve aux Archives photographiques Notman du Musée McCord d'histoire canadienne.

3. Stanley G. Triggs, op. cit., p. 162 (épilogue).

4. Ibid., p. 11 (introduction), p. 166 (appendice), source: Charles F. Notman (1870-1955).

\section{Ibid.}

6. Ibid., aussi communiqué de presse concernant le lancement de la Collection Notman dans les McGill University Museums (12 novembre 1956). 


\section{Les Archives photographiques Notman du Musée McCord}

7. Traduction libre de l'anglais.

8. Lettre de l'Associated Screen News Ltd, datée du 6 mai 1954 et signée par W.J. Singleton.

9. Lettre de l'éditeur Ralph Allen à Alice J. Turnham, datée du 16 décembre 1955.

10. Ibid.; aussi, lettre de Murray Briskin à Alice J. Turnham, datée du 22 décembre 1955.

11. Lettres: A.J. Turnham à R. Allen du Maclean's (30 décembre 1955); A.J. Turnham à William Bentley, administrateur de l'Université McGill (22 février 1956); F. Cyril James, doyen et vice-recteur honoraire de l'Université McGill à Margaret Lukis Lambert, assistante catalogueure de la Collection Notman

(14 juillet 1959) - Monsieur James admet qu'il n'a pas démontré beaucoup d'intérêt envers la problématique de cette collection depuis le début.

12. Lettres de William Bentley à Ralph Allen (18 et 24 juillet 1956).

13. Lettres: Douglas Gowdy, directeur du Maclean's Magazine à Alice J. Turnham (11 mai 1956); réponse de A.J. Turnham à D. Gowdy (28 mai 1956) Ralph Allen (Maclean's) à Alice J. Turnham (4 juin 1956); réponse de A.J. Turnham à R. Allen (12 juin 1956); A.J. Turnham à F. Cyril James (26 juin 1956) - Elle informe le directeur de l'Université qu'elle a reçu deux copies du projet d'offre officielle de la vente. Elle ajoute que les négociations qui se sont échelonnées sur plus de deux ans, "... have at last brought this collection to McGill"; F.C. James à W. Bentley (27 juin 1965) - Il demande à l'administrateur d'apposer sa signature sur les deux copies du projet d'offre officielle de vente, reçues par la directrice des Musées afin d'accélérer l'acquisition.

14. Lettre de Donald N. Byers, Q.C., incluant les copies du projet d'offre officielle de vente à Alice J. Turnham (15 juin 1956).

15. Remarks by Mrs Turnham at reception to mark the acquisition by the McCord Museum of the Notman Photographic Collection! Redpath Museum, November 9, 1956,; aussi "250,000 Photos Span 80 years", The Gazette, November 10, 1956.
16. Lettre de Max Campbell, M.P., d'Ottawa à l'éditeur du Maclean's Magazine (14 octobre 1957), envoyée à Barbara M. Chadwick, catalogueure de la Collection Notman, par Pierre Berton (17 octobre 1957).

17. Lettre de Pierre Berton, éditeur général du Maclean's Magazine à Alice J. Turnham (13 novembre 1957) soulignant que "The National Films Board has expressed an interest in making a picture about Notman collection (...)"; Lettre de A.J. Turnham à Ralph Allen (14 janvier 1959) - "...New York television studio interested in arranging for use of 4 or 5 Notman photographs weekly for 26 weeks on a forthcoming TV panel series." aussi télégramme de Pierre Berton (14 janvier 1958), mentionnant que l'éditeur du Vancouver Sun (section: mode) visitera Alice J. Turnham relativement aux photographies de William Notman.

18. Lettre de Alice J. Turnham à Ralph Allen (10 octobre 1957): une conférence pour le "Women's Art Group" de l'Art Association, le 22 octobre, l'autre pour le "Women's University Club", le 19 novembre; deux lettres de Lloyd Bloom de la Commercial \& Press Photographers' Association of Canada à Toronto: la première datée du 16 décembre 1959 pour proposer la conférence, la deuxième datée du 2 août 1960 pour conclure les arrangements - la conférence a eu lieu le 12 septembre.

19. Press release, Notman Photographic Exibition, Redpath Museum, McGill University, For release Tuesday, May 14, 1957; aussi "Making Victorian Montreal Very Real", The Gazette, May 15, 1957.

20. Press Release, op. cit.; aussi, liste intitulée "Items received from Notman Studio for use in Exhibition", préparée le 15 avril (1957) au musée Redpath, probablement par John A. Kennerley, catalogueur de la "Notman Photograph collection", notes manuscrites présumément écrites par A.J. Turnham.

21. Lettre de Alice J. Turnham à Pierre Berton (17 mai 1957). Cette sorte de collaboration a déjà été établie pour l'occasion du "Press Party" organisé presque simultanément à Montréal (le 9 novembre 1956) et à Toronto (le 12 novembre 1956) pour célébrer l'acquisition de la Collection Notman. Dans sa lettre du 3 octobre 1956, Pierre Berton informe A.J. Turnham 
que dans le tirage du 24 novembre, Maclean's Magazine consacrera 13 pages en plus de la page couverture à la Collection. On peut imaginer ici une sorte de campagne publicitaire. Aussi, dans la même lettre, il propose son aide pour la préparation de la première exposition. Monsieur Berton signale ainsi son intérêt et sa volonté relativement à la promotion. Cette collection photographique leur permet d'offrir un soutien visant à organiser les expositions itinérantes au gré de Alice J. Turnham. Également, il signale la découverte d'objets provenant du Studio Notman, dans la section référence de la Bibliothèque du Royal Ontario Museum. Selon lui, la revendication est possible afin d'ajouter ces objets "...to the collection at McGill."

\section{Press Release, op. cit.}

23. Paul Carle, Alain Mongeau, "La difficile naissance d'une muséologie scientifique moderne. Le cas de l'Université McGill et du Musée Redpath pendant la première moitié du XX siècle", Musées, Vol. 11, Nos 1 et 2, 1988, pp. 6-10; aussi, Paul Carle, Madeleine Dufresne, Lynne Teather, "Le Musée Redpath de 1940 à 1970: les années Johannsen", même source, p. 11-14; Paul Carle, Madeleine Dufresne, Alain Mongeau, Lynne Teather, "Le mouvement de modernisation des musées scientifiques au XX siècle: le cas du Musée Redpath de l'Université McGill", Fontanus, Vol. III, 1990 , pp. 77, 81-82, 86, 89-93, 98-101, 107 (note 69).

24. "It was made clear at that meeting that, in its present quarters, the McCord Museum could accomodate only 300 to 400 albums and that other temporary space would have to be found for the 500,000 plates until the irrelevant items could be discarded. (...) (3) that further investigations be made regarding desirability of microfilming the glass plates and eventual discard of the originals. "; aussi, Lettre de A.J. Turnham à Martha Shepard, National Library of Canada, Ottawa (22 février 1956) - "The question has come up as to the desirability of microfilming some at least of these negatives as a means of reducing the bulk."; lettre de A.J. Turnham au Général Oscar N. Solbert, directeur du George Eastman House of Photography, Rochester, New York (22 février 1956): "When we come to evaluate the collection, some of these negatives undoubtedly will prove historically worthless and can be discarded but we estimate that there will remain a good one hundred thousand glass slides." - Si l'on considère que le nombre estimé de négatifs au début était de 500,000 , sa méthode de calcul est alarmante. Il est difficile d'imaginer que $80 \%$ de cette collection, composée d'objets uniques, peut n'avoir aucune valeur. Aussi, comme on peut lire dans la réponse du 27 mars 1956 du conservateur Beaumont Newhall, de la George Eastman House: "At any rate, I don't feel at the present time that microfilm-reduced negatives will give results at all comparable to larger sized negatives. The ideal thing would be to make duplicate negatives(...)."

25. Témoignage oral de Nora Hague, assistante du conservateur de la photographie des Archives Photographiques Notman, Musée McCord (automne 1991).

26. Lettre de l'Associated Screen News Ltd., datée du 26 janvier 1956; lettre de Alice J. Turnham à F. Cyril James, datée du 3 février 1956.

27. Lettre de George Rance à Alice J. Turnham (18 mars 1957).

28. Lettre de A.J. Turnham à G.E. Worth, The Pestroy Company Ltd. (19 mars 1957).

29. Lettre de Alice J. Turnham à F. Cyril James $(20$ janvier 1956); aussi, lettre de Alice J. Turnham à R.H. Smith, vice-président et directeur général de Morgan Storage and Van Lines Ltd. (16 avril 1957).

30. Lettres de: A.J. Turnham à F,C. James (3 février 1956); F. C. James à A. J. Turnham (7 février 1956); A.J. Turham à M. Birskin (9 février 1956); A.J. Turnham à R. Allen (10 février 1956); A.J. Turnham à M. Briskin (23 février 1956); A.J. Turnham à M. Briskin ( 26 avril 1956); A.J. Turnham à F.C. James (19 juin 1956).

31. Lettres de : A. J. Turnham à M. Briskin ( 9 février 1956); A.J. Turnham à R. Allen (10 février 1956); A.J. Turnham à R. Allen (19 juin 1956), op. cit.

32. Lettres de: A.J. Turnham à Ralph Allen (4 septembre 1956); Pierre Berton à Anne E. McKim (14 novembre 1956). 
33. Time Sheet for Notman Removal (21 mars-11 avril 1957); aussi, lettre de F.C. James à A.J. Turnham (23 juillet 1959); copie de la lettre de Margaret Lukis Lambert à Alice J. Turnham (27 juillet 1959).

34. Lettres de: Pierre Berton à Barbara M. Chadwick (17 octobre 1957); Barbara M. Chadwick à Pierre Berton (4 novembre 1957); Jack Olsen, photo-éditeur du Maclean's Magazine à Barbara M. Chadwick (21 janvier 1958) Barbara M. Chadwick à Jack Olsen (23 janvier 1958); rapport de fréquence sur les demandes, préparé par Barbara $\mathrm{M}$. Chadwick à la fin de l'année 1958; lettre de recommandation de Alice J. Turnham (14 janvier 1960) écrite à la demande de Barbara M. Chadwick.

35. Lettres de: Margaret L. Lambert à Ralph Allen (17 septembre 1958); Ralph Allen à Margaret L. Lambert (23 septembre 1958).

36. Témoignage oral de Nora Hague (autome 1991).

37. Lettre de l'assistant au contrôleur de l'Université McGill, R.L. Puxley à l'agence d'assurances Lewis, Apedaile \& Hanson Inc. (26 septembre 1956); aussi, lettres de A.J. Turnham à Ralph Allen (23 novembre 1956); Anne E. McKim à Pierre Berton (7 février 1957); A.J. Turnham à R.L. Puxley (11 février 1957); Ruth Hertzman (Maclean's Magazine) à Barbara M. Chadwick (22 novembre 1957 et 11 août 1958); Jeanne Paddock (secrétaire éditoriale) à B.M. Chadwick (22 novernbre 1958); B.M. Chadwick à Ralph Allen (7 avril 1959).

38. Témoignage oral de Nora Hague; aussi, rapport de fréquence sur les demandes, préparé par Barbara $M$. Chadwick, op. cit.

39. Report on cellulose acetate folders du 5 décembre 1956, préparé par John Kennerley, Musée Redpath; aussi, lettres de Alice J. Turnham à la Canadian Kodak Sales Company de Toronto (13 décembre 1956); réponse de la Canadian Kodak Sales Limited à A.J. Turnham (19 décembre 1956); témoignage oral de Nora Hague (automne 1991).

40. Copie de la lettre de Margaret Lukis Lambert à Alice J. Turnham (27 juillet 1959), op. cit.; aussi, témoignage oral de Nora Hague (automne 1991).
41. Lettre de R.V.V. Nicolls, Associate Professor, Department of Chemistry, McGill University, à Margaret Lukis Lambert ( 14 juillet 1959); F. Cyril James à M.L. Lambert (23 juillet 1959); (copie) M.L. Lambert à A.J. Turnham (27 juillet 1959), op. cit.; G.L. Long, historian, The Bell Telephone Company of Canada, à A.J. Turnham (15 octobre 1959); G.L. Long à Joseph A. Patterson, Director, The American Association of Museums, Smitbsonian Institution (18 mars 1959); J.A. Patterson à G.L. Long (27 mars 1959); E.E. Howell, American Association of Museums, à G.L. Long (24 avril 1959); Gerhard R. Lomer à A.J. Turnham (19 octobre 1959).

42. Lettre de Alice J. Turnham à F. Cyril James (29 octobre 1959) - "Weighing all the evidence, the Committee was of the unanimous opinion that although lamination offers a remarkable and, as far as can be ascertained safe method for protecting documents and photographs, the problems iaherent in preserving and rendering accessible the 300,000 photographs in the Notman Collection will be more easily, more economically and more quickly served by continuing the present method of cutting the pages in half, inserting them in individual acetate folders and filing them chronologically in vertical steel files".

43. Même lettre, $o p$. cit.

44. Ibid.

45. Témoignage oral de Nora Hague (automne 1991).

46. Rapport de voyage à Ottawa du conservateur Stanley G. Triggs relatant ses visites des collections suivantes: Public Archives of Canada, Picture Division; National Film Board, Still Photo Library; Geological Survey Collection, Department of Mines \& Technology; Department of Agriculture Collection (16 au août 1966).

47. Témoignage oral de Nora Hague (automne 1991).

48. Dane Lanken, "Remarkable exhibit of Notman photographs", The Gazette, novembre 1971; Adrian J. Gatrill, "Portrait of a portraitist", Montreal Star, novembre 1971. 


\section{Les Archives photographiques Notman du Musée McCord}

49. Lettre de H.R. Smith, Morgan Storage and Moving Ltd., à S.G. Triggs (18 mars 1968).

50. Lettre de Isabel M. Dobell, conservatrice en chef du Musée McCord, à Alice E. Johannsen, directrice des University McGill Museums (24 octobre 1968).

51. McCord, Vol. 3, No 2: Hiver 1989-1990, bulletin du Musée McCord d'histoire canadienne; "Le Musée McCord", (dossier), Chantiers, Vol. 8, No 7, septembre 1991, pp.27-28; Guy Boulizon, Les musées du Québec, tome 1: Montréal et l'ouest du Québec, Fides, 1976, p. 74 (Musée McCord); Dusty Vineberg, "The McCord Museum", Montreal Scene, February 17, 1973, pp. 14-15.

52. Dusty Vineberg, op. cit., p. 14.

53. Adrian J. Gatrill, op. cit.; Dane Lanken, op. cit.

54. Canadian Horizons Program, Notification of grant.

55. Lettre de Alice J. Turnham à Niels Montclair (16 mai et 3 juin 1958); Deed of gift (3 juin 1958); Press Release for Saturday, June 7, 1958; Harold Poitras. Press Photographers Then and Now, The Star (revue), juillet 1958.

56. Documents élaborés par Stanley G. Triggs (13 mai 1975 et 10 décembre 1982).

57. Fait noté au cours de mon expérience comme bénévole aux Archives photographiques Notman.

58. "Lancement du projet d'informatisation", Bulletin de la Société des musées québécois, mai 1991, numéro 4 , p. 1.

59. Entretien avec Nora Hague (décembre 1991).

60. Selon mes prévisions basées sur la situation actuelle, pour enfin connaître le nombre exact des objets présentement aux Archives photographiques Notman, il nous faudra attendre quelque 16 ans, à moins que le Musée McCord puisse employer plus de personnel.

61. Dans la lettre de F. Cyril James du 23 juillet 1959 , envoyée à Alice J. Turnham, Margaret Lukis Lambert est décrite comme une photographe amateur même si elle a travaille au Studio avec Charles Notman; aussi, selon le témoignage oral de Nora Hague, Stanley G. Triggs est le premier photographe professionnel à s'occuper de la Collection Notman.

62. Témoignage oral de Nora Hague; aussi, Véronique Robert, op. cit. 